\title{
Cardiac MRI to Investigate Myocardial Scar and Coronary Venous Anatomy Using a Slow Infusion of Dimeglumine Gadobenate in Patients Undergoing Assessment for Cardiac Resynchronization Therapy
}

\author{
Simon G. Duckett, MRCP, ${ }^{1 *}$ Amedeo Chiribiri, MD, ${ }^{1}$ Matthew R. Ginks, MRCP, ${ }^{1}$ \\ Stephen Sinclair, DCR(R), ${ }^{1}$ Benjamin R. Knowles, MPhys, ${ }^{1}$ Rene Botnar, PhD, ${ }^{1}$ \\ Gerry S. Carr-White, FRCP, ${ }^{2}$ Christopher A. Rinaldi, FRCP, ${ }^{1,2}$ Eike Nagel, PhD, ${ }^{1}$ \\ Reza Razavi, FRCP, ${ }^{1,2}$ and Tobias Schaeffter, PhD $^{1}$
}

\begin{abstract}
Purpose: To evaluate a cardiac MR (CMR) examination with slow infusion of a high-relaxivity contrast agent to visualize coronary venous anatomy (CVA) and myocardial scar in heart failure patients awaiting cardiac resynchronization therapy (CRT).

Materials and Methods: Fourteen patients awaiting CRT (seven ischemic cardiomyopathy (ICM) and seven nonICM) and two with normal LV function underwent CMR on a 1.5 Tesla (T) MR scanner. Dimeglumine-gadobenate was slowly infused. Bolus arrival in the LV was measured by a dynamic electrocardiogram (ECG) -triggered inversion recovery (IR) scan subsequent to starting an ECGtriggered respiratory-navigated three-dimensional (3D) SSFP MR scan with IR preparation to acquire systolic whole-heart anatomy for vein visualization. Delayed contrast-enhanced MR scan was performed to assess myocardial scar. CVA obtained by CMR was compared with Xray venography in 11 patients. CVA and scar were segmented and registered for visual inspection.
\end{abstract}

Results: For all subjects, there was excellent visualization of the CVA. All ICM and one non-ICM patient showed scar. There was excellent correlation between veins seen by CMR and venography.

Conclusion: We have demonstrated that slow infusion protocol of dimeglumine-gadobenate can be used to assess both CVA and myocardial scar in a single MR

${ }^{1}$ King,s College London, BHF Centre, Division of Imaging Sciences, NIHR Biomedical Research Centre at Guy's and St Thomas' NHS Foundation Trust, London, UK.

${ }^{2}$ Department of cardiology, Guy's and St Thomas' Hospitals, Westminster bridge, London, UK.

Contract grant sponsor: European Community's Seventh Framework Programme; Contract grant number: FP7/2007-2013 under grant agreement n. 224495 (euHeart project).

*Address reprint requests to: S.G.D., Division of imaging Sciences, The Rayne Institute, 4th Floor, Lambeth Wing, St Thomas' Hospital, London SE1 7EH. E-mail: simon.duckett@kcl.ac.uk

Received December 10, 2009; Accepted September 9, 2010.

DOI $10.1002 /$ jmri.22387

View this article online at wileyonlinelibrary.com. examination. Furthermore, an image overlay technique has been used to show the relationship of scar to the CVA.

Key Words: CRT; whole heart MRI; coronary vein; late enhancement; Gd-BOPTA

J. Magn. Reson. Imaging 201 1;33:87-95.

(C) 2010 Wiley-Liss, Inc.

KNOWLEDGE OF THE anatomy of the coronary venous vessels is becoming increasingly important in interventional cardiology, particularly with respect to cardiac resynchronization therapy (CRT). CRT is an adjuvant therapy for heart failure patients on maximal pharmacological therapy in New York Heart Association (NYHA) class 3 or 4 (1). CRT involves pacing of both left and right ventricles to restore synchronous contraction and improved myocardial efficiency (2). The left ventricular (LV) lead is most commonly implanted transvenously through the coronary sinus (CS) using a posterior lateral, or lateral coronary vein. Failure to implant an LV lead is up to $12 \%$ in large clinical trials (3). Two reasons for this are marked individual variation in coronary venous anatomy, making it difficult to find a suitable position for the LV lead (4), and leads having unacceptable pacing parameters when implanted in areas of myocardial scar (5). Therefore, information about coronary venous anatomy as well as location of myocardial scar provides extremely useful information for procedure planning $(6,7)$.

Coronary venous anatomy is usually imaged at the time of implant with invasive coronary venography. Limitations of this method are that venous anatomy is determined only at the time of implant and no information is provided regarding scar or viable myocardium. Noninvasive methods for assessment of coronary venous anatomy are developing both with computer tomography (CT) (8) and cardiac MR (CMR) imaging (9-12). 
Multislice CT has been used to visualize coronary veins in three dimensions $(8,13)$, but involves ionizing radiation and nephrotoxic contrast agents. CMR has several advantages over CT as it provides information about LV scar $(14,15)$, dyssynchrony (16) and function (17). CMR has previously been used to assess coronary veins using an intravascular contrast $(10,11)$ or without a contrast agent by using an MTCprepulse (9). Although the use of an intravascular contrast agent has been shown to give excellent CV anatomy (11) very limited information with respect to myocardial scar can be obtained.

A retrospective study (18) evaluated CMR angiograms of 31 patients for the ability to visualize coronary venous anatomy using conventional extravascular contrast agent. This study demonstrated the feasibility of using CMR for assessment of scar and venous anatomy. However the scans were not optimized for visualization of the venous system. Furthermore, the average ejection fraction in the patient group was over 50\%. The clinical relevance is, therefore, limited as heart failure patients requiring CRT have ejection fractions of less than 35\% and often have fast irregular heart rhythms and irregular breathing patterns leading to technical challenges with respect to MR image acquisition.

Recently, the slow infusion of the extravascular contrast agent dimeglumine-gadobenate ([Gd-BOPTA], Bracco Imaging SpA, Milan, Italy) has been proposed for whole-heart coronary artery MRA (19). Studies have shown that Gd-BOPTA can be used for the assessment of myocardial scar and viability $(20,21)$, which were found to be comparable to other gadolinium based contrast agents (22). To our knowledge this agent has not previously been used for the combined assessment of coronary vein and myocardial scar.

In this work, we investigated a CMR examination in patients with heart failure using a slow infusion protocol of a Gd-BOPTA to evaluate the coronary venous anatomy, myocardial scar and left ventricular function. In patients with myocardial scar we assessed the relationship between scar and the coronary veins. We also assessed the potential of CMR to aid implant planning by comparing the imaging data from the occlusive venogram taken at the time of CRT implantation with the CMR venous anatomy.

\section{MATERIALS AND METHODS Study Population}

Fourteen heart failure patients (ejection fraction from transthoracic echocardiography $27 \pm 7.0 \%$ ) having a CMR as part of assessment for CRT implants, along with two patients with normal LV function were recruited. Seven patients had ischaemic cardiomyopathy and seven nonischemic cardiomyopathy (Table 1). Subjects with contraindications to MRI or history of anaphylaxis to contrast agent or glomerular filtration rate of $<30 \mathrm{~mL} / \mathrm{min} / 1.73 \mathrm{~m}^{2}$ were excluded. The local ethics committee approved the study and informed consent was obtained from all patients.
Table 1

Demographics of Heart Failure Patients

\begin{tabular}{ll}
\hline & Heart failure patients \\
\hline Sex & 12 men \\
Age & 2 women \\
Etiology & $59.3 \pm 14.5 \mathrm{yr}$ \\
& 7 Non-ischemic \\
& cardiomyopathy \\
Weight & 7 Ischemic cardiomyopathy \\
Average NYHA class & $86.0 \pm 12.5 \mathrm{~kg}$ \\
Heart rate during MRI scan & $2.9 \pm 0.4$ \\
Rhythm & $66.8 \pm 9.3$ bpm \\
& 10 in sinus rhythm \\
& 3 in sinus rhythm with \\
& ventricular ectopics \\
Ejection fraction(trans & 1 in atrial fibrillation \\
thoracic ultrasound) & $27 \pm 7.0 \%$ \\
Septal lateral delay (ms) & $65 \pm 31$ ms $(7 \mathrm{pt}$ \\
& delay $>65$ ms) \\
\hline
\end{tabular}

\section{Data Acquisition}

The patients were scanned using 1.5 Tesla (T) MR scanner (Achieva, Philips Healthcare, Best, Netherlands) with a 32-element cardiac coil (12 patients) and a 5-element cardiac coil (4 large patients due to claustrophobia). Cardiac synchronization was performed with vector electrocardiography (VECG). After localization and a coil sensitivity reference scan an interactive real-time scan was performed to determine the geometry of the short axis (SA), four $(4 \mathrm{CH})$, three $(3 \mathrm{CH})$, and two chamber $(2 \mathrm{CH})$. A multiple slice (M2D) cine steady state free precession (cine-SSFP) scan was performed in SA orientation to assess the ventricular function (fractional anisotropy $[\mathrm{FA}]=60^{\circ}$, repetition time/echo time $[\mathrm{TR} / \mathrm{TE}]=2.9 / 1.5 \mathrm{~ms}$, resolution 2.2 $\times 2.2 \times 10 \mathrm{~mm}, 30$ heart phases). The $4 \mathrm{CH}, 3 \mathrm{CH}$, and $2 \mathrm{CH}$ views were used to assess LV function for regional wall motion abnormalities. Furthermore, visual assessment of the $3 \mathrm{CH}\left(\mathrm{FA}=60^{\circ}, \mathrm{TR} / \mathrm{TE}=3.0 / 1.5\right.$ $\mathrm{ms}$, resolution $2.5 \times 2.5 \times 10 \mathrm{~mm}, 60$ heart phases) view was used to determine timing of the end systole. For the contrast enhanced coronary vein scan, GdBOPTA (dose of $0.1 \mathrm{mmol} / \mathrm{kg}$ ) was slowly infused at a rate of $0.3 \mathrm{~mL} / \mathrm{s}$ with subsequent saline flushing as proposed by Bi et al (19) for coronary arteries. GdBOPTA was used to exploit the high relaxivity ( $\mathrm{r} 1=$ $9.7(\mathrm{mmol} / \mathrm{L})^{-1} \mathrm{~s}^{-1}$ at $\left.1.5 \mathrm{~T}\right)$ and the weak interaction of the contrast agent (CA) with serum albumin.

To determine the optimal start point of the whole heart coronary vein MR scan, a dynamic ECG-triggered two-dimensional (2D) scan with inversion recovery (IR) preparation (TI $=300 \mathrm{~ms}$ ) was used. For coronary vein visualization, an ECG-triggered respiratory navigated 3D IR-SSFP MR scan was applied to acquire the whole-heart during a short interval (60-80 ms) in end systole using a centric profile order and the following parameter: $\mathrm{FA}=50^{\circ}, \mathrm{TI}=300 \mathrm{~ms}$, TR/TE $=$ 4.25/1.44 ms, SENSE $=3-4$ (with SENSE $=2 \mathrm{AP}$ direction and SENSE $=1.5-2$ in RL direction), resolution $1.5 \times 1.5 \times 2 \mathrm{~mm}$ (average number of slices $180 \pm 15)$. The overall scan time for the protocol was 

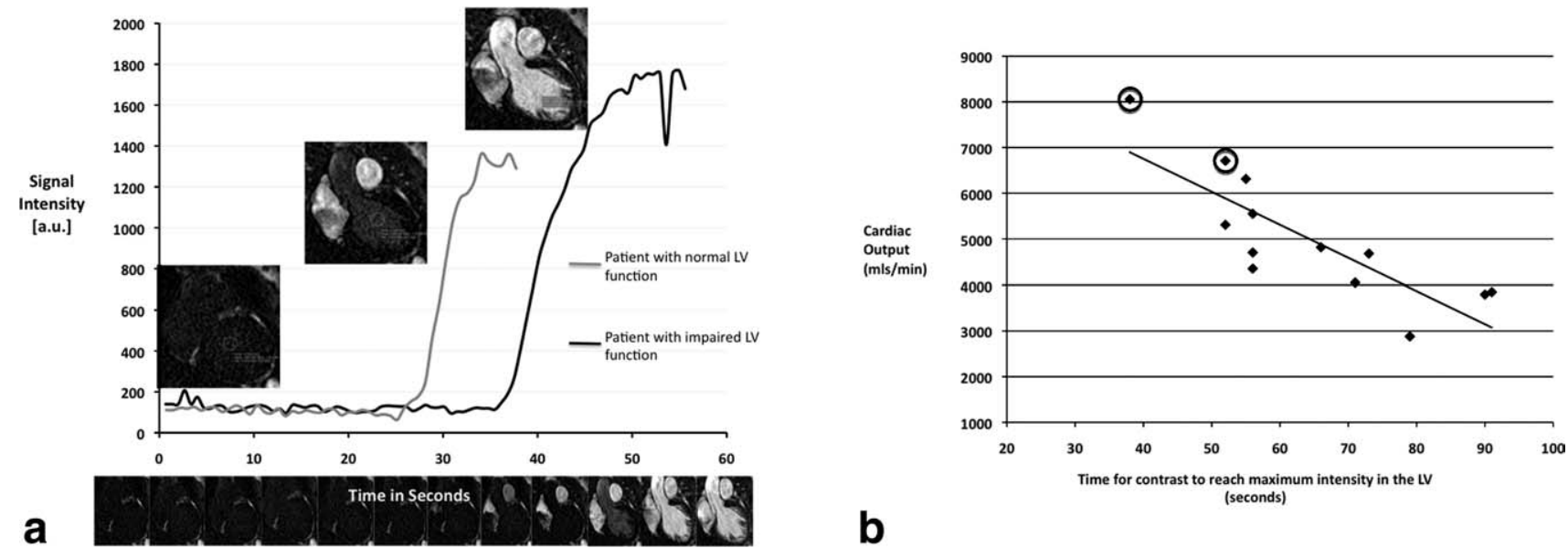

Figure 1. a: Time difference for contrast to reach maximal signal intensity within the LV in a patient with normal and impaired LV function. b: Correlation between cardiac output and time for contrast to reach maximum signal intensity in the left ventricle. The two circled points indicate the patients with normal LV function.

3.5 min (assuming a 100\% respiratory gating efficacy). After the coronary vein scan a delayed contrast-enhanced multi-slice IR gradient echo sequence $\left(\mathrm{FA}=25^{\circ}\right.$, TR/TE $=5.8 / 2.0 \mathrm{~ms}$ ) was performed at end systole to depict areas of scar. A preceding Look-Locker sequence was used to determine the optimal inversion time TI (320 \pm $22 \mathrm{~ms}$ ) to null the signal of the myocardium.

\section{Data Analysis}

$\mathrm{LV}$ volumes were derived from the M2D-cine SSFP data by manually drawing endocardial borders (Philips, Viewforum). Papillary muscles were excluded and end-diastolic volumes (EDV) and end-systolic volume (ESV) were calculated for the left ventricle (LV), from which the ejection fraction and cardiac output (CO) were derived. The arrival of contrast agent bolus in LV was determined from dynamic ECG-triggered IR scan. A linear regression analysis was performed between the time taken for contrast to reach maximum signal intensity for the different cardiac output (Fig. 1).

Volume-rendering of the whole-heart coronary vein scan (3D IR-SSFP) was performed (Philips, Viewforum) to identify the CS and its tributaries. The ostium of the CS was defined as the site where the CS makes an angle with the right atrium. The origin of the great cardiac vein (GCV) was considered immediately after the bifurcation of the posterior vein of the left ventricle (PVLV) when visible or after the posterior interventricular vein in the other subjects. Cardiac veins were classified according to Ortale et al and adopted terminology of Jongbloed et al $(8,23)$.

In all the patients with late enhancement two clinical experts using the standard American Heart Association (AHA) 17 segment model reviewed the position and transmurality of the scar. To determine the relationship between scar and veins we co-registered the 3D whole heart images with the scar imaging using Osirix software (24). For this, we superimposed a cast of the LV volume from the 3D whole heart image with the veins on the scar image. To further visualize the coronary venous anatomy and position in relation to myocardial scar we manually segmented the $3 \mathrm{D}$ whole heart images with ITK-SNAP software (25) to form high fidelity models. The scar was manually segmented using Osirix software and then registered to the 3D segmentation of the left ventricle using geometry information stored in the DICOM header. Once registered, scar segmentation images were projected onto the LV segmentation to provide $3 \mathrm{D}$ visualization of the scar geometry. Using all this information, we then determined the number of veins that were related to areas of scar.

In patients that went onto have a CRT implant at our institute $(n=11)$, we compared the occlusive venogram with the CMR imaging to determined the correlation between the two imaging techniques and to determine if the vein used for final LV lead position was seen on the CMR imaging.

\section{Quantitative Analysis}

Multiplanar reformatting was used to determine the size of the ostium of the CS in anterioposterior and superioinferior direction and to measure the starting diameter of each tributary. The distance between the ventricular tributaries was measured from the volume rendered reconstructions (Fig. 2).

The signal to noise ratio was measured by drawing a region of interest (ROI) in the CS to measure mean signal intensity and two areas in the background define background noise. Because a SENSE acquisition was used, the image noise is spatially varying and usually highly suppressed outside the body. Therefore, two ROI inside the lung were used to define background noise.

Image quality of $3 \mathrm{D}$ coronary vein images was graded using an image quality score of 0 to 4 . $0=$ $\mathrm{CS} / \mathrm{GCV}$ not visible, $1=\mathrm{CS} / \mathrm{GCV}$ visible with markedly blurred borders or edges, $2=\mathrm{CS} / \mathrm{GCV}$ visible with moderately blurred borders or edges, $3=\mathrm{CS} /$ GCV visible with mildly blurred borders or edges, $4=$ $\mathrm{CS} / \mathrm{GCV}$ visible with sharply defined borders or edges (26). For inter observer variability two experienced independent observers analyzed the data sets. 




Figure 2. a,b: Whole-heart 3D reconstruction with the coronary sinus (CS) and tributaries. Also seen are two saphenous vein graphs (SVG). c: An example of the distance between the origin of the CS and the left marginal vein (LMV) and also the angle the LMV makes with the great cardiac vein (GCV). d: The measurement of the diameter of the CS in various plans. LV, left ventricle; LA, left atrium; AIV, anterior inter-ventricular vein.

\section{RESULTS}

The ejection fraction calculated from M2D-cine SSFP for heart failure patients was $27 \pm 9.7 \%$, End diastolic volume was $308.4 \pm 140.4 \mathrm{~mL}(157 \pm 60 \mathrm{~mL} /$ $\mathrm{m}^{2}$ ) and cardiac output was $4.6 \pm 0.9 \mathrm{~L} / \mathrm{min}$. Twelve of the patients went onto have a CRT implant, one of those implants was performed at another institute and in another patient it was not possible to implant an LV lead due to inability to find a stable lead position. Of the two that did not go on to have implants, one patient, although referred with an ejection fraction of $35 \%$ from their transthoracic echocardiogram, was found at MRI assessment to have an ejection fraction of $55 \%$, and one was too unwell to have an implant.

The time taken for the contrast agent to reach the LV was $71.1 \pm 27.2 \mathrm{~s}$ (Fig. 1a). In one patient with severe LV impairment, the bolus track sequence was completed before the contrast reached the left ventricle (maximum duration of bolus track sequence 120 seconds). The time taken for contrast to reach maximum signal intensity and cardiac output were correlated with $r=0.82$ (Fig. 1b).

The scan-time for the ECG-triggered respiratory navigated 3D IR-SSFP MR scan varied within all 16 patients due to different respiratory gating efficiencies. In 13 patients the scan time was $12.8 \pm 3.2 \mathrm{~min}$ (gating efficiency $29 \pm 7 \%$ ) whereas there was a very low gating efficiency (12-15\%) in 3 patients resulting in long scan times up to $30 \mathrm{~min}$.

\section{Anatomical Observations}

The CS and GCV were visualized (Table 2) in all subjects with example reformatted images and segmentations given in Figures 3 and 4. A posterior inter-ventricular (PIV) and left marginal vein (LMV) was seen in 12 patients $(75 \%)$, posterior vein of the LV (PVLV) in 8 patients $(50 \%)$ and an anterior inter-ventricular vein (AIV) in 11 patients (69\%). The mean ostial diameter of the CS and coronary vein branches as well as the mean distance from the ostium of the CS to the various tributaries is shown is Table 3 .

\section{Scar Imaging}

Seven patients with ischemic cardiomyopathy had late enhancement (Figs. 3 and 4) and one of the nonischemic patients had late enhancement attributed to

Table 2

Anatomical Observations and the Correlation With Vein Anatomy During Occlusive Venography

\begin{tabular}{lccccc}
\hline \multicolumn{1}{c}{ Vessel seen } & $\begin{array}{c}\text { No. of vessels } \\
\text { seen by CMR } \\
\text { for all } \\
\text { patients }(\mathrm{n}=16)\end{array}$ & $\begin{array}{c}\text { No. of vessels } \\
\text { seen by } \\
\text { venography for CRT } \\
\text { patients }(\mathrm{n}=11)\end{array}$ & $\begin{array}{c}\text { No. of vessels } \\
\text { seen by CMR } \\
\text { for CRT } \\
\text { patients }(\mathrm{n}=11)\end{array}$ & $\begin{array}{c}\% \text { of vessels } \\
\text { seen by CMR } \\
\text { compared } \\
\text { to venogram }\end{array}$ \\
\hline Coronary sinus & 16 & $100 \%$ & 11 & 11 & 11 \\
Great cardiac vein & 16 & $100 \%$ & 11 & 8 & $100 \%$ \\
Posterior Inter-ventricular vein & 12 & $75 \%$ & 8 & 7 & $100 \%$ \\
Posterior vein of the LV & 8 & $50 \%$ & 8 & 8 & $100 \%$ \\
Left marginal vein & 12 & $75 \%$ & 9 & 7 & $100 \%$ \\
Anterior inter-ventricular vein & 11 & $69 \%$ & 2 & 2 & $78 \%$ \\
Additional lateral veins & 4 & $25 \%$ & 0 & 0 & $100 \%$ \\
Additional posterior veins & 1 & $6 \%$ & & & N/A \\
\hline
\end{tabular}




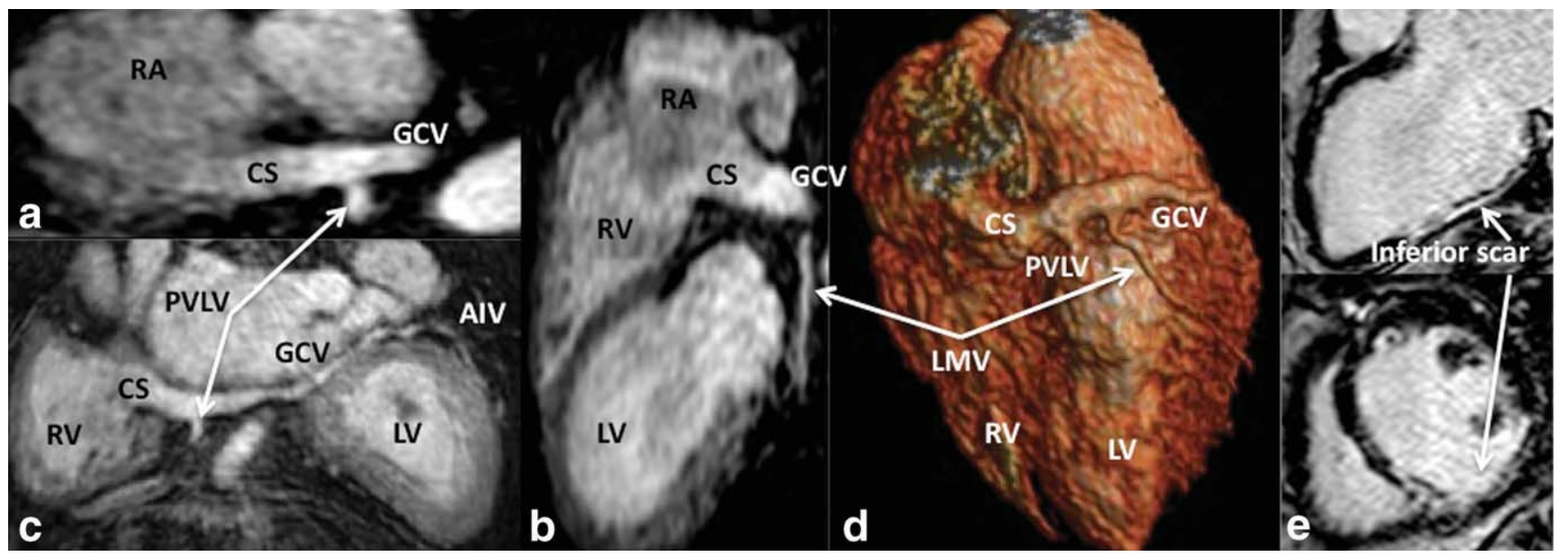

Figure 3. a,b: Multiplanar reformatted (MPR) images of the coronary sinus (CS) and great cardiac vein (GCV) with the posterior lateral vein of the LV (PVLV). c: MPR image showing the extent of the coronary vein from the CS to the anterior inter-ventricular (AIV). d: A 3D reconstruction of the coronary venous system with the various branches. e: Late enhancement images showing inferior scar. RA, right atrium; LMV, left marginal vein; RV, right ventricle; LV, left ventricle.

an embolic event. Of the patients with scar, one had anterior scar, two anterior/lateral scar, one anterior/ septal scar, one lateral scar and three inferior scar. For six of the patients with scar we were able to attribute at least one coronary vein to an area of scar (Figs. 5 and 6) (Table 4).

\section{Comparison of CMR Venous Anatomy and Venography at Implantation}

In all 11 patients that had implants at our institute, there was 100\% correlation between the CS, GCV, PIV, PVLV, LMV, and additional lateral veins seen on CMR compared with the occlusive venography (Fig. 5). In two of the patients, the AIV was not seen with CMR imaging, but was visible at venography. In all of the patients, the vein used for final LV lead placement was visualized (Table 2).

\section{Image Quality}

The average score for the CS was 3.0 and GCV 2.6 with a good agreement between two observers. With respect to the vessels seen the observers agreed for in all cases for the CS, GCV, and PIV. Observer 1 identi- fied an AIV vein that observer 2 did not and also two additional lateral veins. The average signal to noise ratio for all patients was 19.5.

\section{DISCUSSION}

To our knowledge, this is the first study to demonstrate that CMR can be used to accurately assess function, coronary venous anatomy and myocardial scar in a group of patients with heart failure and dilated ventricles awaiting cardiac resynchronization therapy in a single MR examination. We have shown that this CMR protocol can be used to delineate the coronary venous anatomy and correlates well to the occlusive venography. Due to the resolution of the 3D whole heart image $(1.5 \times 1.5 \times 2 \mathrm{~mm})$, the venogram gives more detailed information about small vessels and the distal parts of coronary vein branches; however, the CMR imaging provides an accurate non invasive assessment of the CS and main tributaries. When comparing the CMR venous anatomy to the venogram in only two patients we were unable to see main vessels, which were visible on the venogram. In both patients, the vessel was the AIV, which, is not

Table 3

Ostial Diameter of the Identified Vein and the Distance From the Ostium of the Coronary Sinus to the Identified Vein and Measurable Vessel Length

\begin{tabular}{lccccc}
\hline $\begin{array}{c}\text { CS } \\
\text { tributary }\end{array}$ & $\begin{array}{c}\text { Ostial diameter of } \\
\text { identified vein } \\
\text { Superior/inferior }(\mathrm{mm})\end{array}$ & $\begin{array}{c}\text { Ostial diameter of } \\
\text { identified vein } \\
\text { Anterior/posterior }(\mathrm{mm})\end{array}$ & $\begin{array}{c}\text { Average angle between } \\
\text { the tributary and the } \\
\text { CS or GCV }\end{array}$ & $\begin{array}{c}\text { Average distance from } \\
\text { the ostium of the } \\
\text { CS }(\mathrm{mm})\end{array}$ & $\begin{array}{c}\text { Average length of } \\
\text { the identified } \\
\text { vein }(\mathrm{mm})\end{array}$ \\
\hline CS & $12.5 \pm 4.1$ & $13.9 \pm 5.7$ & $\mathrm{~N} / \mathrm{A}$ & $143.8 \pm 50.0^{\mathrm{b}}$ & $\mathrm{N} / \mathrm{A}$ \\
PIV & $5.0 \pm 0.8$ & $4.7 \pm 1.2$ & $74.7^{\circ} \pm 27.6^{\circ}$ & $12.0 \pm 4.3$ & $35.8 \pm 13.6$ \\
PVLV & $5.4 \pm 1.5$ & $5.4 \pm 1.0$ & $108.3^{\circ} \pm 30.7^{\circ}$ & $32.6 \pm 16.4$ & $42.0 \pm 34.3$ \\
LMV & $4.8 \pm 1.0^{\mathrm{a}}$ & $4.7 \pm 1.2^{\mathrm{a}}$ & $99.1^{\circ} \pm 32.0^{\circ}$ & $68.8 \pm 19.4$ & $33.3 \pm 23.5$ \\
AIV & $3.2^{\circ} \pm 1.0^{\mathrm{a}}$ & $3.1 \pm 0.8^{\mathrm{a}}$ & $119.6^{\circ} \pm 28.7^{\circ}$ & $146.5 \pm 27.6$ & $23.9 \pm 16.1$ \\
\hline
\end{tabular}

annable to accurately measure in 2 patients.

${ }^{\mathrm{b}}$ Total length of vessel measurable (length from coronary sinus to end of anterior intra-ventricular vein).CS = coronary sinus; GCV = great coronary vein; PIV = posterior inter-ventricular vein; PVLV = posterior vein of the LV; LMV = left marginal vein; AIV = anterior interventricular vein. 


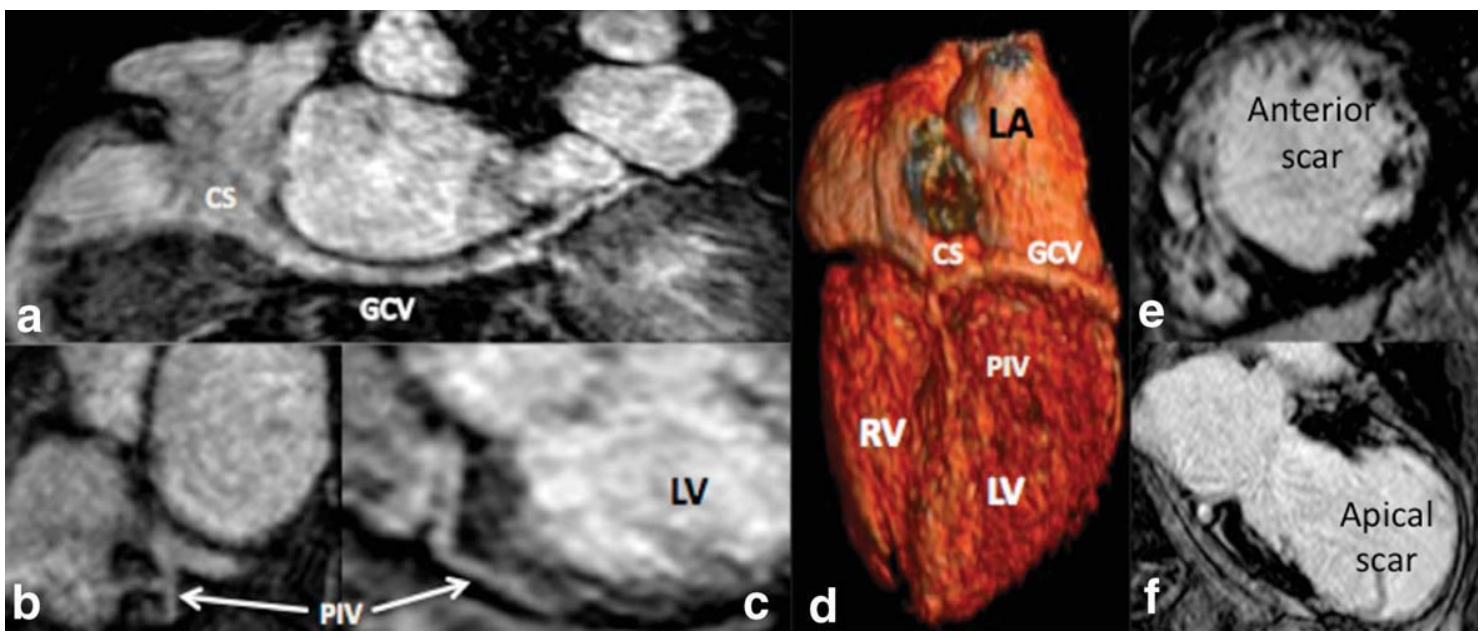

Figure 4. a-c: Multiplanar reformatted images of the coronary sinus (CS) and the great cardiac vein (GCV). d: A 3D reconstruction of the coronary venous system. e,f: Late enhancement images with extensive anterior apical scar. LA, left atrium; $\mathrm{RV}$, right ventricle; LV, left ventricle; PIV, posterior inter-ventricular vein.

normally a target for LV lead placement in CRT. Furthermore, using multiplanar reconstructions and volume rendered images we were able to measure the diameter of the CS ostium and the distance potential target vessels are from the CS ostium as well as the angle of take off from the GCV. This information can be valuable in CRT procedure planning. When an occlusive venogram is performed the balloon is blown up some distance from the CS ostium and then contrast is injection. Veins between the CS ostium and the balloon are often poorly filled by contrast and it is possible to miss potential target vessels. The ability to show potential targets vessels are present and their size and distance from the CS before the procedure means that these vessels are less likely to be missed by a suboptimal venogram.

In all patients that underwent CRT, the target vessels for LV placement were visualized by CMR as were all the veins for final lead position. Furthermore, by overlaying segmented coronary vein anatomy and scar we were able to determine which veins were in areas of scar and transmurality of the scar. Using this technique has the potential to guide implanters before the procedure on the coronary venous anatomy and the potential of which veins may be related to scar.

Previous studies have used volunteers or patients principally with normal LV size and function (10$12,18)$. In this study, we have demonstrated that it is feasible to provide clinically relevant information in patients with heart failure. This cohort of patient is technically more challenging to scan, as there are often problems with arrhythmias. In our study, one patient was in atrial fibrillation and a further three had multiple ventricular ectopics. Of interest, the patient with atrial fibrillation, although having a long scan time, had good image quality graded as 3 by both observers for the CS and the GCV. Patients also have difficulty lying flat for long periods due to the nature of heart failure symptoms. This often leads to irregular breathing patterns and, therefore, difficulty with the navigator efficiency prolonging scan times. All of the patients with heart failure had intra-

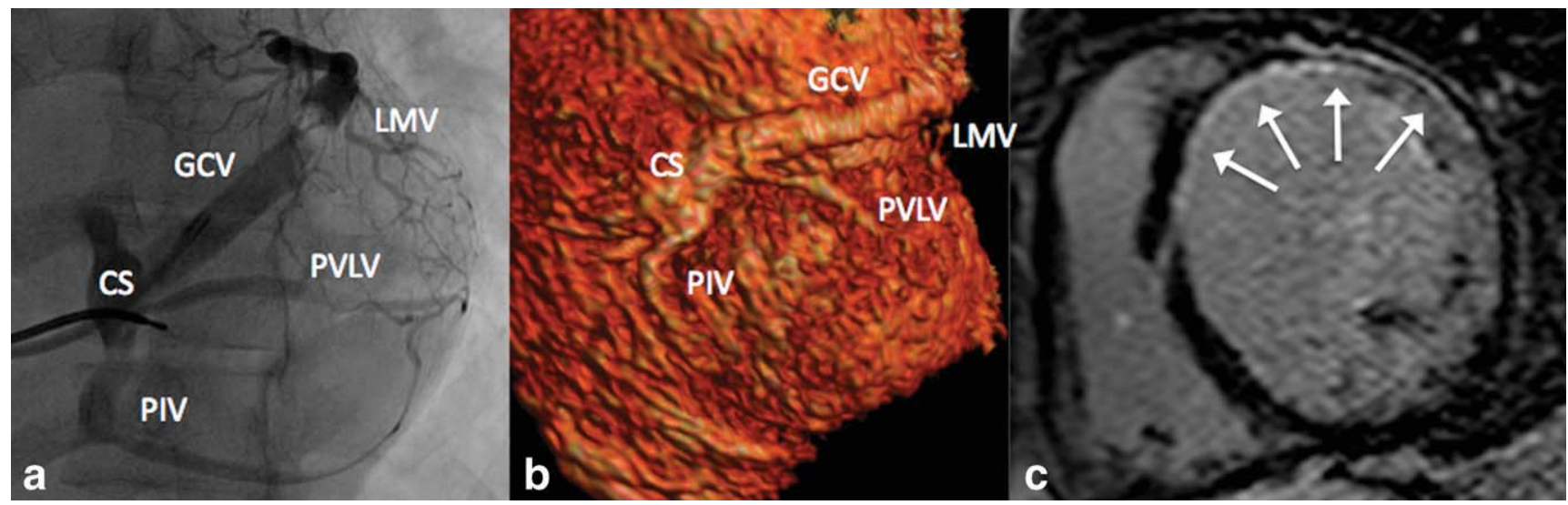

Figure 5. Shows a patient with a dilated cardiomyopathy and anterior scar. a: Occlusive venogram performed at time of CRT implant. b: Volume rendered 3D reconstruction of the heart and coronary veins showing the correlation with the occlusive venogram. c: Mid-ventricular short axis late gadolinium enhanced image showing subendocardial anterior scar of 50 to $75 \%$ transmurality. 

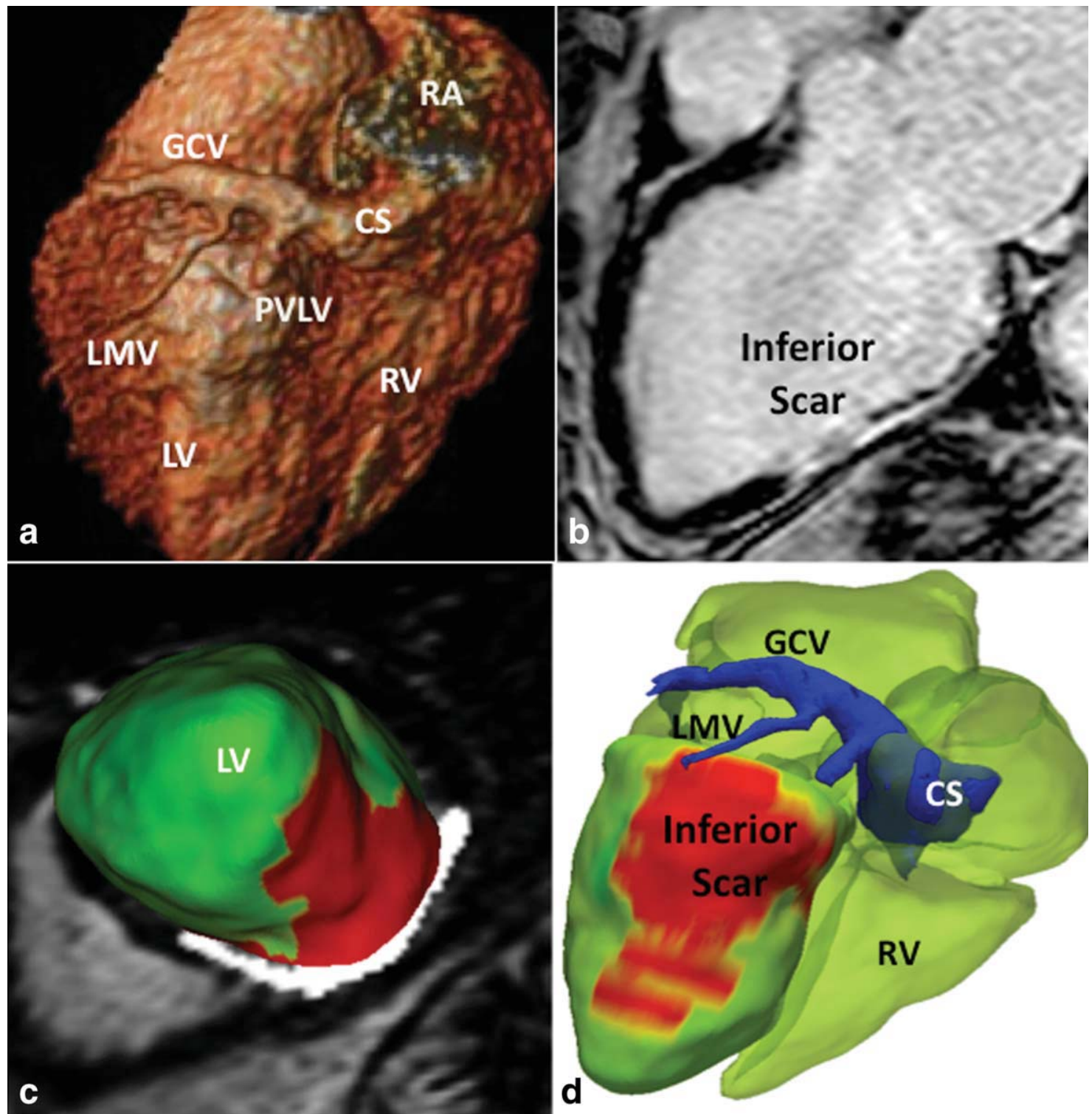

Figure 6. a: A $3 \mathrm{D}$ reconstruction of the heart with the coronary venous system. b: Two chamber multislice delayed contrastenhanced MR scan showing inferior late enhancement c: Segmented LV registered to the scar imaging in the short axis view. The scar has been manually segmented and is imposed on the segmented LV shown as red in the image. d: Segmented whole heart with the coronary veins and scar all superimposed. RA, right atrium; LMV, left marginal vein; RV, right ventricle; LV, left ventricle; GCV, great cardiac vein; PVLV, posterior lateral vein of the LV.

ventricular conduction delay with significant ventricular dyssynchrony. The 3D whole heart scan was triggered during end systole because although the diastolic quiescent period is longer the area of the CS is larger during the systolic quiescent period making imaging easier (9). We have used a narrow acquisition window of 60 to $90 \mathrm{~ms}$. Although this increased the scan duration, it was important to ensure image quality. Our scan times were long due to both irregular heart rates and breathing patterns as well as short acquisition windows. The scan would benefit from new innovations that improve the respiratory gating efficiency.

Due to poor cardiac output of heart failure patients there is marked variation in the time for contrast agent to disseminate within the blood pool and reach the coronary veins compared with subjects with normal LV function. Using a dynamic ECG-triggered IR scan to measure bolus arrival in the LV we were able to optimize the timing of the 3D IR-SSFP scan (Fig. 1a). In one of the patients with a nonischemic cardiomyopathy the bolus track sequence had finished before contrast entering the LV cavity. This emphasizes the importance of the correctly timing the start of the $3 \mathrm{D}$ whole heart scan with centric profile order when using a slow infusion protocol.

Scar imaging was done at the same timing as the 3D IR recovery whole heart acquisition (end systole). This allowed direct comparison between the scar imaging, segmented left ventricle and coronary veins. However, in both scans two different respiratory motion compensation strategies were applied, that is, 
Table 4

Position, Transmurality of Scar, and Number of Veins Associated With Scar

\begin{tabular}{|c|c|c|c|c|c|c|}
\hline Case vo. & Etiology & Scar area & $\begin{array}{l}\text { AHA segments } \\
\text { involved }\end{array}$ & $\%$ Scar thickness & $\%$ Scar vol & $\begin{array}{c}\text { No. of veins } \\
\text { associated with scar }\end{array}$ \\
\hline 1 & ICM & Inferior, basal/mid & $3,4,5,9,10,11$ & $75-100 \%$ & $14 \%$ & $2^{a}$ \\
\hline 2 & ICM & Inferior basal/mid & $4,5,10,11$ & $50-100 \%$ & $8 \%$ & 1 \\
\hline 4 & $\mathrm{ICM}$ & Anterior/septal/lateral ${ }^{b}$ & $\begin{array}{c}1,2,6,7,8,12,13 \\
14,15,16,17\end{array}$ & $\begin{array}{l}\text { Basal } / \text { mid } 25-50 \% \\
\text { Apex } 100 \%\end{array}$ & $36 \%$ & 1 \\
\hline 5 & $\mathrm{ICM}$ & $\begin{array}{l}\text { Anterior/septal/lateral } \\
\text { Mid/apical }\end{array}$ & $\begin{array}{c}2,3,7,8,9,12,13 \\
14,15,16,17\end{array}$ & $100 \%$ & $55 \%$ & 0 \\
\hline 7 & ICM & $\begin{array}{l}\text { Anterior/septum } \\
\text { Mid/apical Apex }\end{array}$ & $7,8,9,12,13,14,16,17$ & $100 \% 100 \%$ & $23 \%$ & 0 \\
\hline 9 & ICM & $\begin{array}{l}\text { Anterior/septum } \\
\text { Lateral Apex }\end{array}$ & $\begin{array}{c}5,6,7,8,10,11,12 \\
13,14,15,17\end{array}$ & $75-100 \% 75-100 \% 100 \%$ & $27 \%$ & 1 \\
\hline 10 & DCM & Anterior & $1,2,6,7,8,12$ & $75-100 \%$ & $12 \%$ & 1 \\
\hline 15 & ICM & Inferior/lateral & $4,5,10,11$ & $25-50 \%$ & $14 \%$ & 1 \\
\hline
\end{tabular}

Images for this patient seen in Figure 6.

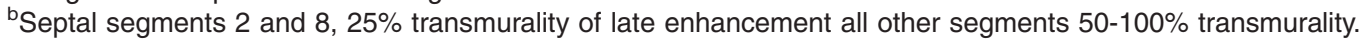

$\mathrm{ICM}=$ ischemic cardiomyopathy; DCM = dilated cardiomyopathy; AHA, American Heart Association.

a respiratory navigator and breathhold. Two points in the papillary muscles were used as anatomical landmarks to briefly investigate the misalignment between the coronary vein and the late enhancement scan. We found relatively small displacements of $3 \pm 3.5 \mathrm{~mm}$ in 14 patients and larger displacements of up to $20 \mathrm{~mm}$ in two patients. In future, the registration between both scans can be improved by using the same respiratory navigator.

We have looked at a small number of heart failure patients $(\mathrm{n}=14)$ and now need to get greater numbers in particular with ischemic heart disease to allow further assessment of the relationship of the coronary veins to the myocardial scar. We have not compared our technique with other noninvasive imaging modalities or extra vascular contrast agents. However, our results with respect to vessels seen and measurements of length and diameter are comparable to previous studies, which involved volunteers or patients with normal LV function.

We used a slow infusion to increase the circulating time for the contrast agent and improve imaging of the venous anatomy. Due to the time taken to acquire the 3D whole heart scan it may be more appropriate for a bolus to be given and further work needs to be done to optimize the methods for contrast infusion. Furthermore, we have used a constant inversion time for the coronary vein scan, because a determination of TI using a Look-Locker scan before would delay the start 3D IR-SSFP MR scan with centric profile order. The use of a constant TI and the different heart rate of the patients resulted in different image contrast among the patients.

As 11 of the patients went onto have devices we were able to show that the CMR imaging compared favorably to the venogram anatomy. Although we were able to determine which vessels were present ideally we would have liked to compare the measurements made at CMR with the venogram anatomy. However, for a quantitative assessment of the size and length of the different territories numerous X-ray views would be necessary which would increase the procedure time and radiation dose to the patient.
In conclusion, we have demonstrated that CMR can delineate the coronary venous anatomy and its relationship to myocardial scar in this patient population. This has been helpful for procedure planning and during device implantation using the segmented volumes together with overlaying software (27). Although these techniques are in their infancy, there is the potential to guide lead placement to avoid areas of scar and to reduce procedure time and exposure to contrast agents as well as radiation. With evidence demonstrating the clinical importance of these factors in CRT outcomes, the use of this information may become a prerequisite in CMR assessment of heart failure.

\section{ACKNOWLEDGMENTS}

The authors thank John Totman and Yingliang Ma for their support in the preparation of this study.

\section{REFERENCES}

1. Cleland JG, Daubert JC, Erdmann E, et al. The effect of cardiac resynchronization on morbidity and mortality in heart failure. N Engl J Med 2005;352:1539-1549.

2. Bank AJ, Kaufman CL, Kelly AS, et al. Results of the Prospective Minnesota Study of ECHO/TDI in Cardiac Resynchronization Therapy (PROMISE-CRT) study. J Card Fail 2009;15:401-409.

3. Abraham WT, Hayes DL. Cardiac resynchronization therapy for heart failure. Circulation 2003;108:2596-2603.

4. Kautzner J, Riedlbauchova L, Cihak R, Bytesník J, Vancura V. Technical aspects of implantation of LV lead for cardiac resynchronization therapy in chronic heart failure. Pacing Clin Electrophysiol 2004;27:783-790.

5. Bleeker GB, Kaandorp TA, Lamb HJ, et al. Effect of posterolateral scar tissue on clinical and echocardiographic improvement after cardiac resynchronization therapy. Circulation 2006;113:969-976.

6. Ypenburg C, Schalij MJ, Bleeker GB, et al. Impact of viability and scar tissue on response to cardiac resynchronization therapy in ischaemic heart failure patients. Eur Heart J 2007;28:33-41.

7. Rovner A, de Las Fuentes L, Faddis MN, Gleva MJ, Davila-Roman VG, Waggoner AD. Relation of left ventricular lead placement in cardiac resynchronization therapy to left ventricular reverse remodeling and to diastolic dyssynchrony. Am J Cardiol 2007;99:239-241.

8. Jongbloed MR, Lamb HJ, Bax JJ, et al. Noninvasive visualization of the cardiac venous system using multislice computed tomography. J Am Coll Cardiol 2005;45:749-753.

9. Nezafat R, Han Y, Peters DC, et al. Coronary magnetic resonance vein imaging: imaging contrast, sequence, and timing. Magn Reson Med 2007;58:1196-1206. 
10. Rasche V, Binner L, Cavagna F, et al. Whole-heart coronary vein imaging: a comparison between non-contrast-agent- and contrast-agent-enhanced visualization of the coronary venous system. Magn Reson Med 2007;57:1019-1026.

11. Chiribiri A, Kelle S, Gotze S, et al. Visualization of the cardiac venous system using cardiac magnetic resonance. Am J Cardiol 2008;101:407-412.

12. Chiribiri A, Kelle S, Kohler U, et al. Magnetic resonance cardiac vein imaging: relation to mitral valve annulus and left circumflex coronary artery. JACC Cardiovasc Imaging 2008;1:729-738.

13. Gerber TC, Sheedy PF, Bell MR, et al. Evaluation of the coronary venous system using electron beam computed tomography. Int $\mathrm{J}$ Cardiovasc Imaging 2001;17:65-75.

14. Kim RJ, Wu E, Rafael A, et al. The use of contrast-enhanced magnetic resonance imaging to identify reversible myocardial dysfunction. N Engl J Med 2000;343:1445-1453.

15. Orn S, Manhenke C, Anand IS, et al. Effect of left ventricular scar size, location, and transmurality on left ventricular remodeling with healed myocardial infarction. Am J Cardiol 2007;99: 1109-1114.

16. Bilchick KC, Dimaano V, Wu KC, et al. Cardiac magnetic resonance assessment of dyssynchrony and myocardial scar predicts function class improvement following cardiac resynchronization therapy. JACC Cardiovasc Imaging 2008;1:561-568.

17. Nagel E, Schneider U, Schalla S, et al. Magnetic resonance realtime imaging for the evaluation of left ventricular function. J Cardiovasc Magn Reson 2000;2:7-14.

18. Younger JF, Plein S, Crean A, Ball SG, Greenwood JP. Visualization of coronary venous anatomy by cardiovascular magnetic resonance. J Cardiovasc Magn Reson 2009;11:26.
19. Bi X, Carr JC, Li D. Whole-heart coronary magnetic resonance angiography at 3 Tesla in 5 minutes with slow infusion of GdBOPTA, a high-relaxivity clinical contrast agent. Magn Reson Med 2007;58:1-7.

20. Andersen K, Hennersdorf M, Cohnen M, Blondin D, Modder U, Poll LW. Myocardial delayed contrast enhancement in patients with arterial hypertension: initial results of cardiac MRI. Eur J Radiol 2009;71:75-81.

21. Sandstede JJ, Beer M, Lipke C, et al. Time course of contrast enhancement patterns after Gd-BOPTA in correlation to myocardial infarction and viability: a feasibility study. J Magn Reson Imaging 2001;14:789-794.

22. Schlosser T, Hunold P, Herborn CU, et al. Myocardial infarct: depiction with contrast-enhanced MR imaging--comparison of gadopentetate and gadobenate. Radiology 2005;236:1041-1046.

23. Ortale JR, Gabriel EA, Iost C, Marquez CQ. The anatomy of the coronary sinus and its tributaries. Surg Radiol Anat 2001;23: 15-21.

24. Rosset A, Spadola L, Ratib O. OsiriX: an open-source software for navigating in multidimensional DICOM images. J Digit Imaging 2004; 17:205-216.

25. Yushkevich PA, Piven J, Hazlett HC, et al. User-guided 3D active contour segmentation of anatomical structures: significantly improved efficiency and reliability. Neuroimage 2006;31:1116-1128.

26. McConnell MV, Khasgiwala VC, Savord BJ, et al. Comparison of respiratory suppression methods and navigator locations for MR coronary angiography. AJR Am J Roentgenol 1997;168:1369-1375.

27. Rhode KS, Hill DL, Edwards PJ, et al. Registration and tracking to integrate X-ray and MR images in an XMR facility. IEEE Trans Med Imaging 2003;22:1369-1378. 